\title{
Cherenkov light-based beam profiling for ultrarelativistic electron beams
}

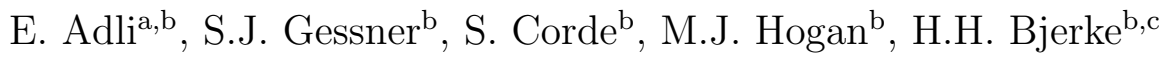 \\ ${ }^{a}$ Department of Physics, University of Oslo, N-0316 Oslo, Norway \\ ${ }^{b}$ SLAC National Accelerator Laboratory, 2575 Sand Hill Road, Menlo Park, California \\ 94025, USA \\ ${ }^{c}$ Department of Physics, Norwegian University of Science and Technology, N-7491 \\ Trondheim, Norway
}

\begin{abstract}
We describe a beam profile monitor design based on Cherenkov light emitted from a charged particle beam in an air gap. The main components of the profile monitor are silicon wafers used to reflect Cherenkov light onto a camera lens system. The design allows for measuring large beam sizes, with large photon yield per beam charge and excellent signal linearity with beam charge. The profile monitor signal is independent of the particle energy for ultrarelativistic particles. Different design and parameter considerations are discussed. A Cherenkov light-based profile monitor has been installed at the FACET User Facility at SLAC. We report on the measured performance of this profile monitor.
\end{abstract}

Keywords: electron beams, profile monitor, Cherenkov light, FACET User Facility 
7

field acceleration [1], laser plasma acceleration [2], and two-beam acceleration machines where drive beams are heavily decelerated with large energy spread, such as CLIC [3].

We present here a beam profile diagnostic method based on Cherenkov light generated by an electron beam traveling through air. The profile monitor works equally well for electrons and positrons. This type of diagnostic was first used for plasma experiments at the SLAC Final Focus Test Beam Facility $[4,5]$ and has been further developed for the spectrometer at the FACET User Facility $[1,6]$, as described in this paper.

The use of Cherenkov radiation for profile monitoring has the significant advantage over the more established use of optical transition radiation (OTR), in that the light yield per beam electron may be much larger. Cherenkov radiation in air generates on the order of 30 photons per electron per meter, in the optical range (shown below). In comparison the OTR energy spectrum, $d W_{\text {otr }} / d \omega$, from relativistic electrons is approximately given by $[7]$

$$
\frac{d W_{\text {Otr }}}{d \omega} \approx 4.9 \times 10^{-37} \ln \gamma
$$

where $\gamma$ is the Lorentz factor, which yields about 0.05 OTR photons per electron per surface unit for ultrarelativistic beams, in the optical range. By using enough path length to generate Cherenkov light, one may easily get a factor 100 stronger signal with a detector based on Cherenkov light compared to an OTR-based setup. For beams with large energy spread, the Cherenkov light has the added advantage over OTR that the light yield is independent of the particle energy. High light yield is important in advanced accelerator experiments where small charge signals may be of great experimental interest, as illustrated for example by the low charge accelerated tail described in [4]. On the other hand, beams in the FACET experimental area may have very high charge densities, reaching on the order of $1000 \mathrm{nC} / \mathrm{mm}^{2}$. The Cherenkov profile monitor has the advantage that the light yield is linear in charge over the full charge density range. Scintillating materials used for beam profiling, for example Kodak Lanex, also provide high light yield. These type of screens, however, may cease to be linear in charge at certain charge densities. In [8] several types of Lanex are reported to have a saturated signal at densities of less than $100 \mathrm{pC} / \mathrm{mm}^{2}$, four orders of magnitude lower than the FACET peak charge density. Large charge density may also damage scintillating materials, while our setup based on Cherenkov radiation has proven resistant to damage, as reported later in this paper. Another 
advantage of the Cherenkov profile monitor is that upstream photons, originating from upstream beam interactions with beam line elements or, in our case, the plasma, will not generate Cherenkov radiation. The Cherenkov profile monitor can therefore separate the incoming electrons from incoming photons. Finally, as we will discuss, our profile monitor design provides the possibility of large field of views, and is a relatively robust and simple system to set up as well as a cost-effective solution for a profile monitor.

We first discuss general principles and parameter considerations. We then describe the design of the Cherenkov light-based profile monitor for the spectrometer for the FACET User Facility and report on its performance.

\section{Principle}

Charged particles traveling faster than the speed of light in a given medium emit Cherenkov radiation [9].

The index of refraction of dry air, $n_{\text {air }}$, can be estimated from the modified Edlén formulas [10,11] and ranges from 1.000270 to 1.000278 , at $20 \mathrm{deg} \mathrm{C}$ temperature and $1 \mathrm{~atm}$. pressure, when the wavelength ranges from $400 \mathrm{~nm}$ to $750 \mathrm{~nm}$. We will in this paper assume typical laboratory conditions where the air humidity, pressure and temperature do not change significantly, and assume the index of refraction to $n_{\text {air }}=1.000274 \pm 0.000004$. We assume thus the speed of light in air, $v_{\text {air }}=c / n_{\text {air }}$, to be constant. Particles with normalized velocity $\beta=v / c$ greater than $1 / n_{\text {air }}$ will emit Cherenkov radiation. For electrons, this corresponds to an energy of $21 \mathrm{MeV}$ or more. The opening angle of the resulting Cherenkov radiation is given by

$$
\cos \theta=\frac{1}{n_{\text {air }} \beta} .
$$

We note that for electrons with energy of $150 \mathrm{MeV}$ or more, the opening angle will be within $1 \%$ of the opening angle for infinite energy,

$$
\theta_{C}=\cos ^{-1}\left(1 / n_{\text {air }}\right)=24.2 \mathrm{mrad} .
$$

In this paper we are mostly concerned with ultrarelativistic electrons and we assume for calculations the opening angle to be that of infinite energy, $\theta_{C}$. For this energy range, the Cherenkov profile monitor has the advantage that the signal intensity is for our purposes independent of the particle energy. 
The Cherenkov radiation frequency spectrum is given by the Frank-Tamm formula [12], which yields the number of photons, $N$, emitted per unit length, $d x$, and wavelength interval, $d \lambda$,

$$
\frac{d^{2} N}{d \lambda d x}=\frac{2 \pi \alpha}{\lambda^{2}} \sin ^{2} \theta_{C},
$$

where $\alpha \approx 1 / 137$ is the fine-structure constant. We are interested in the frequency range corresponding to the sensitive range of the cameras used the experimental setup, which in our case is from about $400 \mathrm{~nm}$ to about 750 nm. Using Eq. (1) we calculate the number of photons per electron per unit length, generated in this frequency range, to

$$
\frac{d N}{d x}=2 \pi \alpha \sin ^{2} \theta_{C} \int_{400 \mathrm{~nm}}^{750 \mathrm{~nm}} \frac{d \lambda}{\lambda^{2}}=29 \mathrm{~m}^{-1} .
$$

In order to control the amount of photons generated, the electron beam to be profiled passes through two wafers as it travels through air. The first wafer blocks light already generated up to this point, and the second wafer reflects the light generated between the two wafers. The total number of photons can be regulated by adjusting the gap between the two wafers, $d_{\text {gap }}$. We require a wafer material that has good reflectivity in the optical range, as well as a high damage threshold. We have chosen silicon, which reflects about $30-40 \%$ of incident light. Silicon wafers have the advantage that they can be procured off-the-shelf in appropriate shapes and thicknesses for a reasonable price.

In order to image the beam, a standard CCD camera and a commercial lens may be used, adjusted to point-to-point focus from the center of the air gap onto the camera sensor. In order to image an electron, the electron position must be in the field of view of the camera and either all light emitted, or a fraction of the light emitted, must hit the camera lens. Figure 1 a) illustrates how the profile monitor can be set up.

\section{Parameter considerations}

We now discuss a few key considerations particular to the Cherenkov profile monitor which are required to optimize the profile monitor performance. 

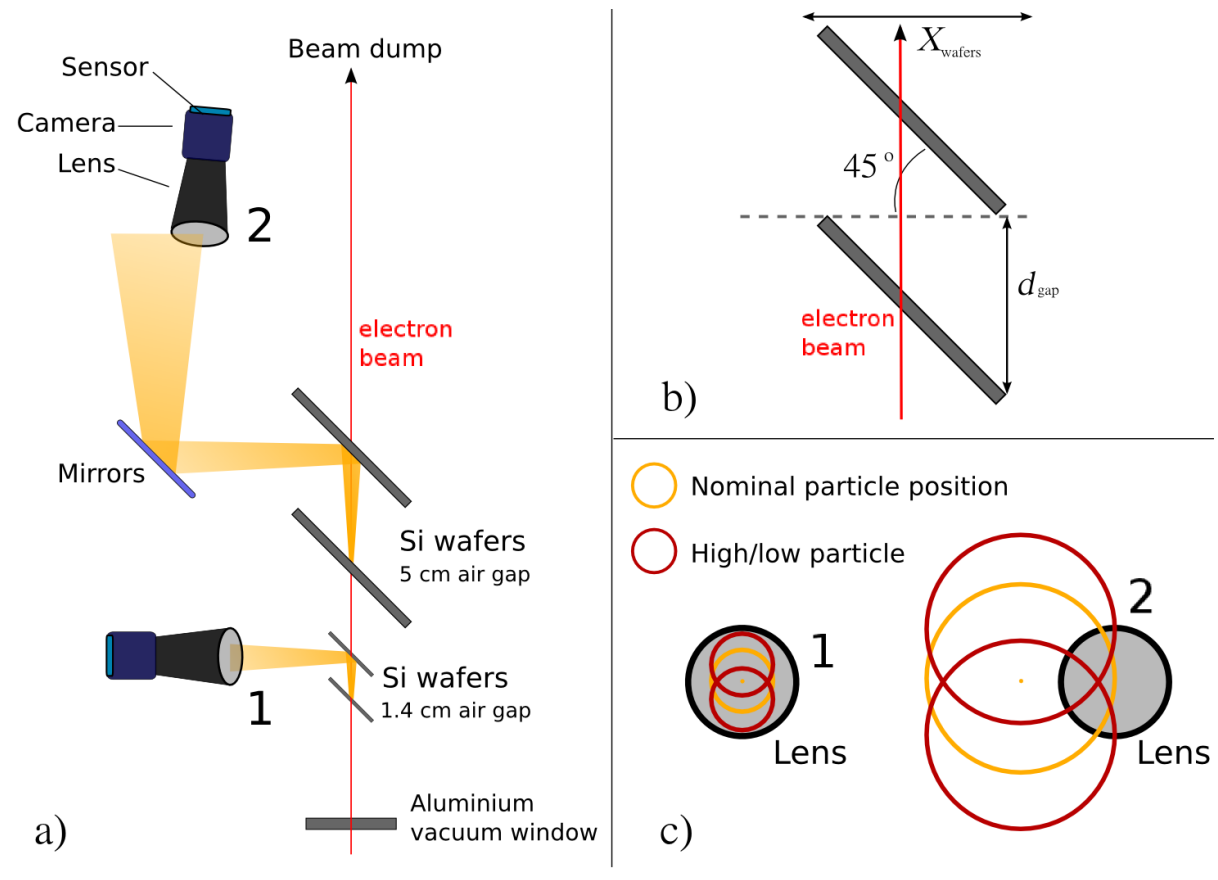

Figure 1: Illustration of a Cherenkov light-based profile monitor setup. An ultrarelativistic electron beam enters air, and emits Cherenkov radiation. A first silicon wafer blocks upstream light, while a second silicon wafer reflects the light generated between the two wafers onto a lens. Apart from scattering in the Si, the profile monitors are non-intrusive and several monitors might be used simultaneously. a) shows a schematic overview of the FACET Cherenkov spectrometer profile monitor setup. Parameters for this setup will be discussed later in the paper. b) shows a possible geometry for the wafer setup. c) shows the principles of the two modes of operation, where either all the Cherenkov light is collected on the lens (marked with "1"), or, only a fraction of the ring is collected on the lens (marked with "2"). 


\subsection{Length of air gap versus resolution limitations}

One contribution to the detector resolution is given by the fact that the Cherenkov light is emitted continuously along a finite air gap. To estimate the resolution limit due to the air gap we use a ray tracing model assuming linear optics. In this model the light emitted from a certain distance upstream or downstream of the focal plane will form a ring on the camera sensor, equivalent to a depth of field circle of confusion. Each longitudinal slice along the air gap give origin to rings with different radii. We assume that the lens is set to focus in the middle of the air gap, at longitudinal position $\mathrm{z}=0$. At the camera sensor, the radius of the rings originating at a longitudinal distance $\pm z$ from the focus are given by $r_{\text {sensor }}(z)=m z \tan \left(\theta_{C}\right)$, where $m$ is the lens magnification. The intensity per pixel of the rings decreases as $1 / r_{\text {sensor }}$ since the same number of photons is spread out over a larger area. In order to compare the depth of field effect with the size of the beam to be imaged, we are interested in the corresponding size of the rings in the focal plane, given by $r(z)=r_{\text {sensor }}(z) / m=z \tan \left(\theta_{C}\right)$. The net effect is that an infinitely thin electron beam will be observed as having a finite thickness. In Figure 2 we calculate this distribution for a $5 \mathrm{~cm}$ long air gap, where the lens focus is in the middle of the gap. The distribution rms width in the focal plane is in this case $155 \mu \mathrm{m}$.

The blocking and the reflecting wafers must be rotated by the same angle in order not to generate correlation between beam transverse position and signal intensity. Placing the wafers at 45 degree angle with respect to the beam trajectory minimizes the length of the air gap with respect to the projected width of the wafers in the plane orthogonal to the beam trajectory. The minimum length of the air gap is therefore given by the projected width of the wafers, $d_{\text {gap }} \geq X_{\text {wafers }}$, as illustrated in Figure 1 b). An important system design trade-off is therefore the resolution limitations originating from the air gap depth-of-field error, versus transverse field of view limitations from the projected wafer width. The air gap may be made larger than the projected wafer width, if it is desired to increase the camera signal level, as the photon yield increases linearly with the air gap.

\subsection{Field of view versus fraction of collected light}

The Cherenkov radiation is propagating in a cone with opening angle $\theta_{C}$. This can be exploited to image a field of view (FOV) much larger than the lens itself, because only a fraction of the Cherenkov ring needs to be captured in order to image the emitting charge. We first discuss the simpler case, where 

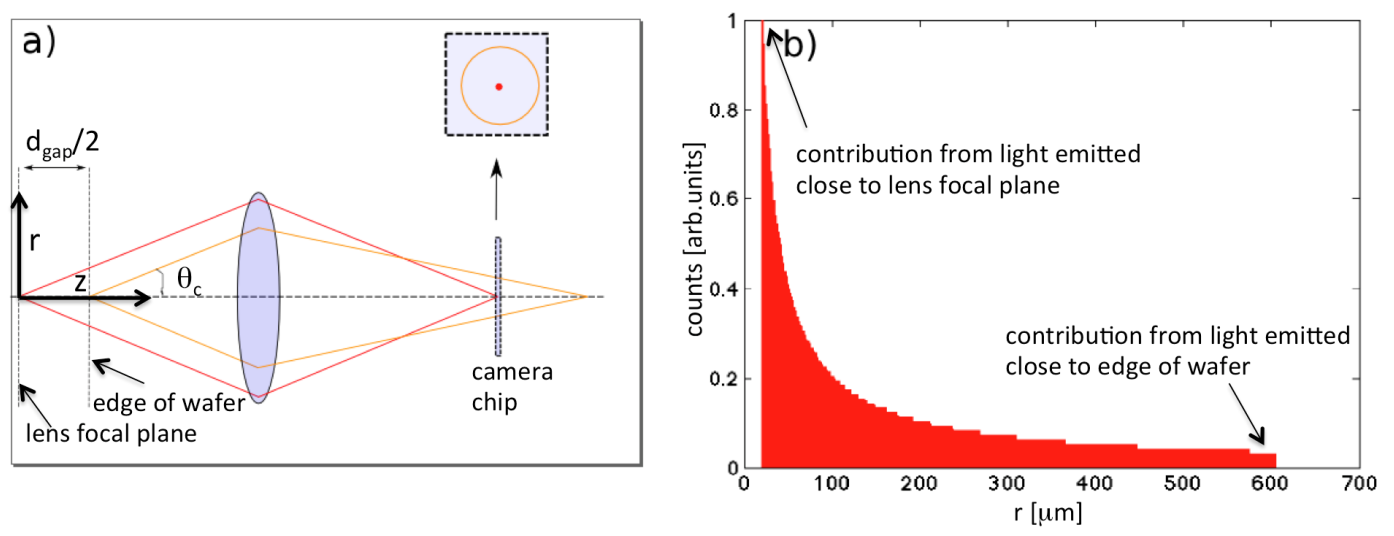

Figure 2: (a) Principle of the depth of field blurring due to Cherenkov light emitted away from the focal plane of the camera system, calculated using a ray tracing model. Photons emitted a distance $\pm z$ away from the focal plane will form rings on the sensor, with the corresponding ring radius in the focal plane given by $r(z)=z \tan \left(\theta_{C}\right)$. (b) The corresponding intensity distribution in the focal plane, after an infinitely thin electron beam has passed through an air gap of $5 \mathrm{~cm}$. Photons originating at the focus in the middle of the air gap are imaged at the center pixel at $r=0$. Photons generated upstream or downstream of the focus are spread out in rings with intensity proportional to $1 / r$ yielding the distribution shown in the figure. The rings with the largest radius originate from light emitted when the electrons pass close to the wafer edge. In the focal plane this corresponds to rings with radius $r=2.5 \mathrm{~cm} \times \tan \left(\theta_{C}\right)=600 \mu \mathrm{m}$. The distribution rms width in the focal plane is in this case $155 \mu \mathrm{m}$. The granularity of this calculation corresponds to the FACET camera pixel size. 
the lens captures the entire ring for the desired FOV, as illustrated in Figure $1 \mathrm{c}) 1$. In this case the Cherenkov light signal is distributed onto the imaging sensor proportional to the electron charge. The maximum effective FOV of the camera is limited to the lens diameter $2 r_{\text {lens }}$ minus the diameter of the Cherenkov ring at the position of the lens, $2 r_{\text {ring }}=2 s \theta_{C}$, where we define $s$ as the distance from the center of the wafers to the lens. In order to image the full FOV, the optical magnification factor, $m=f /(s-f)$, where $f$ is the focal length of the lens, must be $m \leq X /\left(2 r_{\text {lens }}-2 r_{\text {ring }}\right)$, assuming a square sensor with width $X$; see Figure $1 \mathrm{~b}$ ). A smaller magnification would not be useful, as the light would not be collected by the lens. A larger magnification may be useful to increase the resolution, if the depth-of-field error is not a limiting factor, which again depends on the air gap, as discussed above.

As an alternative mode of operation, the profile monitor can be designed such that only a fraction of the Cherenkov ring hits the lens. The corresponding setup is illustrated in Figure 1 c) 2, for a case where the Cherenkov ring radius is several times larger than the lens radius. This mode of operation has the significant advantage that the FOV in one plane can be as large as desired, limited only by wafer sizes and resolution limits. One possible use of this mode of operation is for energy spectrum measurement where the electron beam is highly dispersed according to its energy in one plane. The fraction of the Cherenkov light ring that hits the lens depends on the position of the charge imaged, as illustrated in Figure $1 \mathrm{c}$ ). In order to optimize the system performance we calculate the fraction of light collected for the desired FOV. To find a general expression, we use Figure 3, where $D$ is the distance from the center of the lens to the center of the Cherenkov ring, and $r_{\text {lens }}$ and $r_{\text {ring }}$ are the radii of the lens and the Cherenkov ring respectively.

The intersection of the two rings in Figure 3 along the horizontal axis is calculated as

$$
\xi=\frac{D^{2}-r_{\text {lens }}^{2}+r_{\text {ring }}^{2}}{2 D}
$$

and along the vertical axis as

$$
\psi^{2}=\frac{4 D^{2} r_{\text {ring }}^{2}-\left(D^{2}-r_{\text {lens }}^{2}+r_{\text {ring }}^{2}\right)^{2}}{4 D^{2}} .
$$

The full angle of the Cherenkov ring inside the intersections is given by

$$
\alpha=2 \arctan \frac{\psi}{\xi} \text {. }
$$




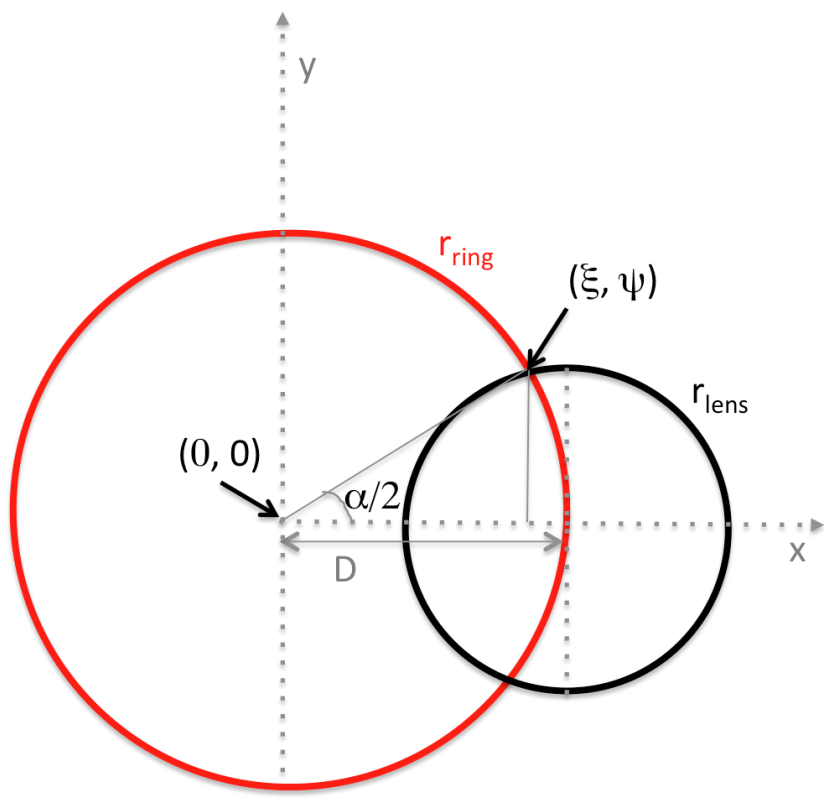

Figure 3: If the camera lens is put far enough from the air gap, the Cherenkov ring (red) will have a larger radius than the radius of the lens (black). The fraction of light falling on the lens and being imaged on the sensor will depend on the horizontal and vertical position of the emitting charge. By optimizing the ring radii and the distance between the Cherenkov ring and the lens, the variation in the fraction of light falling on the lens can be kept relatively small over the entire field of view. 
It follows that the fractional light yield from a particle for which the Cherenkov ring has a center at a transverse distance $D$ from the lens center, is given by

$$
p_{\text {light }}=\frac{\alpha}{2 \pi}=\frac{1}{\pi} \arctan \frac{\sqrt{4 D^{2} r_{\text {ring }}^{2}-\left(D^{2}-r_{\text {lens }}^{2}+r_{\text {ring }}^{2}\right)^{2}}}{D^{2}-r_{\text {lens }}^{2}+r_{\text {ring }}^{2}} .
$$

For charge which at the object plane is offset with respect to the particle with its ring at a distance $D$, the fraction of the light can be calculated by substituting $D$ in Eq. (3) by

$$
D_{\text {charge }}(x, y)=\sqrt{(D-x)^{2}+y^{2}},
$$

where $x$ is the offset in the horizontal dimension and $y$ the offset in the vertical dimension. Eqs. (3) and (4) can be used to calculate the variation in light yield across the camera FOV. Typically, one would like good light yield in the center of the FOV, though there may be less light yield towards the edges of the FOV. We will refer to this effect as "vignetting".

In the limit where the lens is placed very far away from the wafers such that $r_{\text {ring }} \gg r_{\text {lens }}$, and where the Cherenkov ring center is at a distance $D=r_{\text {ring }}$ from the lens center, the light yield can be approximated by

$$
p_{\text {light }}=\frac{1}{\pi} \frac{r_{\text {lens }}}{r_{\text {ring }}} \sqrt{1-x^{2}} .
$$

In this case any imaged particle with zero horizontal position will have the same light yield; the vignetting does not depend on vertical position. The part of the beam which can be imaged is limited only by the camera FOV and the wafer size. In the horizontal plane it falls off when the almost straight lines of the Cherenkov rings move towards the edges of the lens. To achieve constant light yield over a large range, in one dimension, a configuration with the lens far away may thus be used. The focal length of the lens must be comparably large in order to optimize the magnification for the camera sensor size. As an example we illustrate the vignetting effect for the case of $r_{\text {ring }} \gg r_{\text {lens }}$, where a lens with radius $r_{\text {lens }}=7.5 \mathrm{~cm}$ and a focal length of $f=600 \mathrm{~mm}$ is placed a distance $s=20 \mathrm{~m}$ away from the wafer. The Cherenkov rings have a radius of $r_{\text {ring }}=\theta_{C} s=48 \mathrm{~cm}$ at this distance. Figure 4 shows the vignetting effects for this configuration, assuming the distance between the ring and the lens is set to $D=r_{\text {ring }}$, calculated using Eqs. (3) and (4). The FOV shown corresponds to a camera sensor size of $14 \times 17$ 
a)

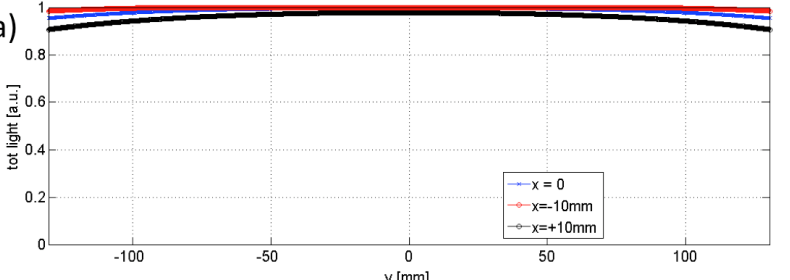

b)

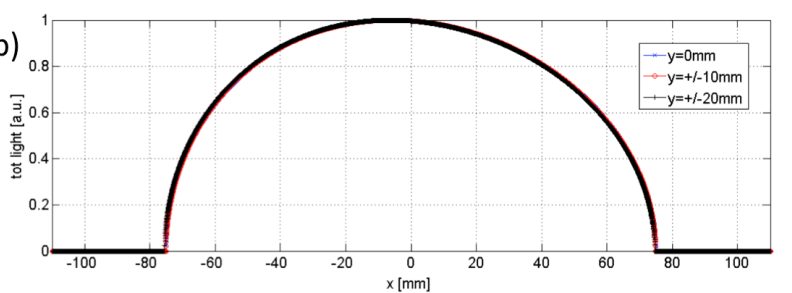

c)

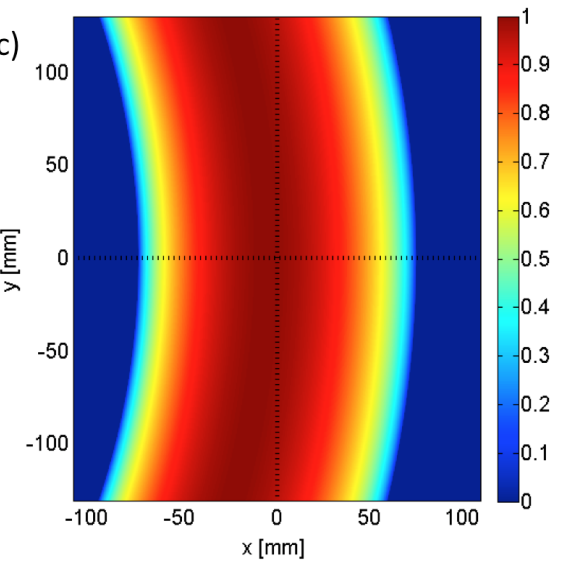

Figure 4: An example calculation of the vignetting effects for the case where the Cherenkov ring radius is larger than the lens radius. The lens has a radius of $7.5 \mathrm{~cm}$ and is placed $20 \mathrm{~m}$ away from the silicon wafers. The resulting Cherenkov ring radius is $48 \mathrm{~cm}$. The figures are calculated using Eqs. (3) and (4). (a) Along the vertical axis, the light yield varies by only up to $\pm 2.5 \%$, over a range of $20 \mathrm{~cm}$. (b) In the horizontal dimension, the light yield follows Eq. (5) closely. (c) A two-dimensional calculation of the light yield as function of both horizontal and vertical charge locations.

$\mathrm{mm}^{2}$, and we have assumed that the wafers cover the entire FOV. We observe that on the horizontal axis the variation in light yield is $\pm 2.5 \%$ up to $y$ values of $\pm 13 \mathrm{~cm}$. On the $y=0$ axis the light yield is limited by the lens radius, and closely follows Eq. (5).

\section{Experimental setup at FACET}

Practical systems will be limited by the lenses and cameras available as well as physical boundaries. We describe here the experimental setup of a Cherenkov light-based profile monitor installed at the FACET User Facility, as part of the FACET imaging spectrometer. This setup is based on the principle of capturing a fraction of the Cherenkov light on the lens in order to access a large field of view in the energy-dispersed plane (vertical plane). The field of view captures particles decelerated to less than half the FACET nominal energy $(20.35 \mathrm{GeV})$ and up to infinite energy. We first discuss the parameters for this system, then we compare calculated resolution and vignetting against measurements. 
The FACET Cherenkov monitor is installed in the FACET tunnel, close to the beam dump, on an optics table with a footprint of $1.2 \times 2.4 \mathrm{~m}^{2}$. A Nikkor lens with radius of $r_{\text {lens }}=7.5 \mathrm{~cm}$ and a focal length of $f=600 \mathrm{~mm}$ is installed on a CMOS camera with a sensor size of $14.0 \times 16.6 \mathrm{~mm}^{2}$. The CMOS camera is of type PCO.edge 5.5, with 16 bit dynamic range [13]. The lens aperture was fully opened, with an f-number of $f_{\#}=f / 2 r_{\text {lens }}=4$. The camera lens was positioned $s=5.9 \mathrm{~m}$ away from the center of the wafers, using three high-quality 6" mirrors. The resulting magnification $m=0.104$ yields a FOV of $13.5 \times 16.0 \mathrm{~cm}^{2}$. The resulting Cherenkov ring at the lens is $r_{\text {ring }}=\theta_{C} s=14.3 \mathrm{~cm}$. The air gap between the wafers is $d_{\text {gap }}=5 \mathrm{~cm}$. The distance from the center of the Cherenkov ring for the nominal energy particle, to the center of the lens, was set to $D=0.84 r_{\text {ring }}=12.0 \mathrm{~cm}$, optimized to give the minimum overall vignetting effects.

In addition to the Cherenkov radiation generated in the air gap, optical transition radiation (OTR) is generated in the transition between the wafers and the air. From [7] we have estimated the OTR emission to about 0.05 OTR photons per electron per surface, which is a few percent of the Cherenkov emission for our experimental setup. The intensity distribution of the transition radiation is very sharply peaked at an angle $\theta=1 / \gamma[7]$, which in our experiment amounts to $\theta=0.049 \mathrm{mrad}$. However, in the experimental setup, the radiation narrowly emitted around this angle is not collected by the camera lens, since the lens is positioned at an offset with respect to the line of sight, $\theta=0$. We therefore expect no contributions from OTR in our profile monitor.

The CMOS camera is sensitive to wavelengths up to about $750 \mathrm{~nm}$, which for $f_{\#}=4$ gives a diffraction limit on the camera sensor of $1.22 \times 750 \mathrm{~nm} \times$ $f_{\#}=3.7 \mu \mathrm{m}$ and a diffraction limit on the target of $35 \mu \mathrm{m}$. In comparison, the resolution of the optical system (CMOS camera + lens) was measured to be $88 \mu \mathrm{m}$ using a 1951 USAF resolution test target [14], which is close to, and possibly dominated by, the pixel resolution of $62 \mu \mathrm{m}$.

The beam enters the air from vacuum through a $5.1 \mathrm{~mm}$ thick aluminum window. The window is relatively thick since it is optimized for a large opening for the beam of $15 \mathrm{~cm}$ in the horizontal plane and $40 \mathrm{~cm}$ in the vertical plane. The rms multiple scattering angle from the window is $143 \mu \mathrm{rad}$ [15]. The Cherenkov wafers are located $86 \mathrm{~cm}$ downstream of the window, yielding a minimum beam spot size at the wafers of $122 \mu \mathrm{m}$. The scatter from the wafers themselves has negligible effect on the minimum spot size. The experimental setup in the FACET tunnel is shown in Figure 5. 


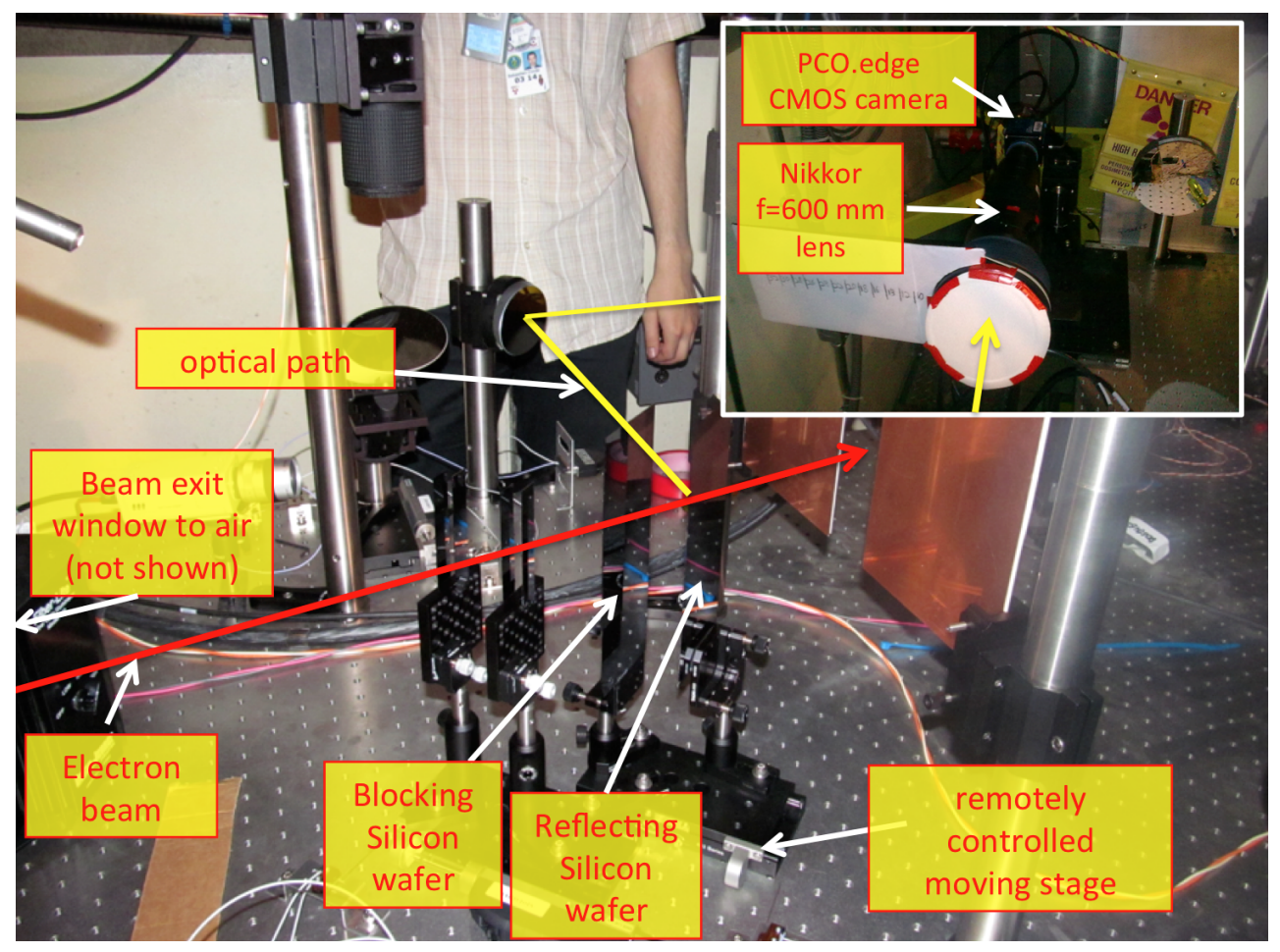

Figure 5: The experimental setup in the FACET tunnel. All components are installed on a $1.2 \mathrm{~m} \times 2.4 \mathrm{~m}$ optical table. The upper right inset depicts components installed in the upper right corner of the main picture. The electron beam, indicated in red, passes through two silicon wafers. The first wafer blocks the light generated upstream, while the second wafer reflects the light generated between the two wafers onto a Nikkor $600 \mathrm{~mm} 15$ $\mathrm{cm}$ diameter lens, reflecting off three mirrors for a total path length of $6 \mathrm{~m}$. Only part of the light path is indicated in the picture. The wafers can be moved in and out of the electron beam line, using a remotely controlled moving stage. In the picture there are also additional equipment related to other diagnostics, not described here. 


\section{Performance}

We have tested the experimental setup described above, using the FACET electron beam with a nominal charge of $2 \times 10^{10} e$ and a nominal energy of $20.35 \mathrm{GeV}$.

\subsection{Sensitivity and linearity}

Each camera pixel has a range of $2^{16}$ output values. We correlated the camera signal level with the charge in the spectrometer line, measured using a BPM. This yielded a sensitivity of 52 electrons or positrons per pixel output value, corresponding to 75 photons per pixel output value, using Eq. (2). The measurements were performed with relatively new wafers. In comparison, the effective noise level of the system as installed in the FACET tunnel is about 5 bits (a value of 31). Thus, about 1600 electrons or positrons per pixel is required to generate a signal above the noise level. The camera is fitted with a retractable filter that decreases the sensitivity by a factor 70 , in order to avoid camera sensor saturation when the FACET beam has a small energy spread. The measurements showed that the signal level is linear in charge density, as expected. The data for the sensitivity calculation is shown in Figure 6.

\subsection{Vignetting}

The FACET spectrometer profile monitor is optimized for a large field of view, and for small vignetting effects. The large radius lens in combination with the large distance between the silicon wafers and the lens leads to small vignetting effects in the field of view, according to the ray tracing model, as shown in Figure 4. We have experimentally measured the light yield in a region of $2.4 \mathrm{~cm}$ in the horizontal by $5 \mathrm{~cm}$ in the vertical, and compared it to the theoretical values predicted from Eq. (3). The measurements were performed by moving the beam using correctors and dipole magnets in a $5 \times$ 5 grid. Within the range documented here, the beam could be moved without inducing significant losses during the transport to the dump. Figure 7 (top row) shows the predicted values to the left, and the measured values to the right. For the measured results, the nominal beam is centered on $x=0$ and $y=0$. In both cases the light yield is within a few percent of the maximum, showing that the vignetting effects in the profile monitor are indeed small. In comparison, we also measured the vignetting with the lens iris partially 


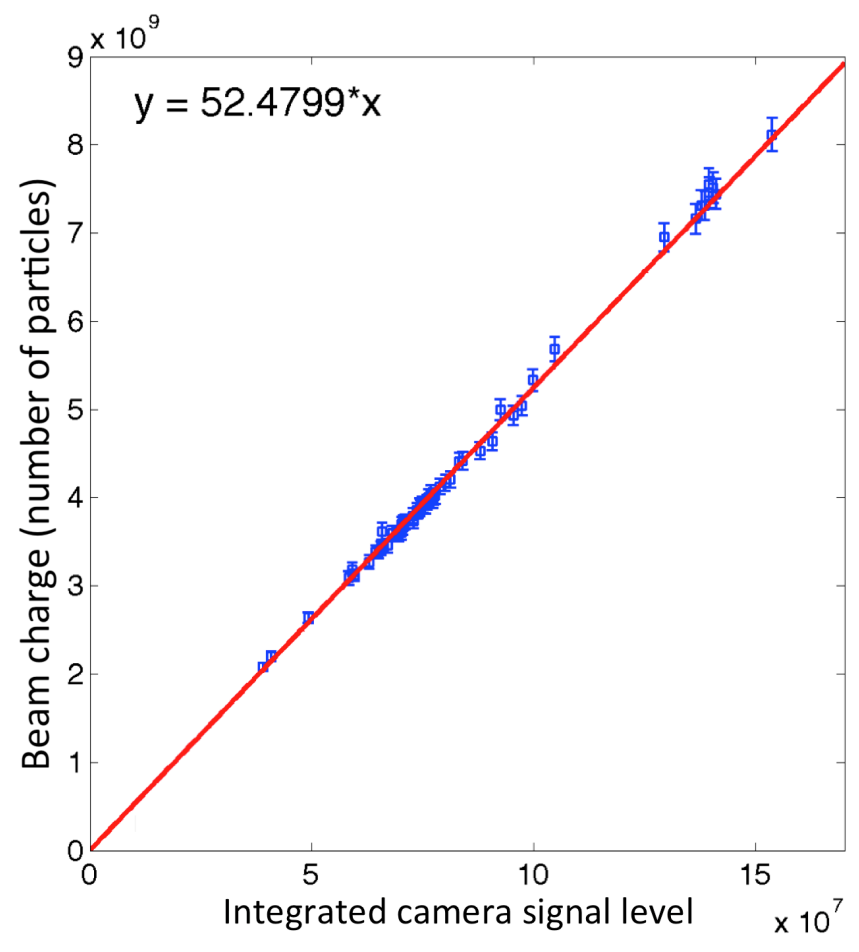

Figure 6: Integrated camera signal level correlated with beam position monitor (BPM) signal level, for a beam of up to $8 \times 10^{9}$ positrons. The BPM signal shown is the mean value of three different BPMs upstream of the profile monitor. The error bar represents the standard deviation of the three BPM readings. The measurement shows the linearity of the profile monitor signal versus beam charge, and corresponds to a charge calibration of 52 positrons per pixel output value. 
closed, corresponding to an aperture of $5 \mathrm{~cm}$. as opposed to $15 \mathrm{~cm}$ with the iris fully open. Figure 7 (bottom row) shows the predicted vignetting to the left, and the measured vignetting to the right. We see that there is reasonable correspondence between the predicted and measured fall-off, verifying the assumptions of the Cherenkov ring propagation used in our system description.

\subsection{Resolution}

By adding the resolution contributions from known sources, we estimate a minimum resolution of

$$
\sigma_{\text {res, th }}=\sqrt{\sigma_{\mathrm{sc}, \mathrm{Alu}}^{2}+\sigma_{\mathrm{sc}, \mathrm{Si}}^{2}+\sigma_{\mathrm{dof}}^{2}+\sigma_{\mathrm{opt}}^{2}}=216 \mu \mathrm{m}
$$

where $\sigma_{\mathrm{sc}, \mathrm{Alu}}=122 \mu \mathrm{m}$ is due to scattering of the beam exit window into air; $\sigma_{\mathrm{sc}, \mathrm{Si}}=11 \mu \mathrm{m}$ is due to scattering in upstream wafers of another dump table diagnostics; $\sigma_{\text {dof }}=155 \mu \mathrm{m}$ is the depth of field error due to the air gap, calculated following Figure $2 ; \sigma_{\mathrm{opt}}=88 \mu \mathrm{m}$ is the optical resolution measured with the calibration target. These contributions to the resolution are equal for both the horizontal and the vertical plane. In the horizontal plane there is an additional contribution to the resolution because the lens collects light at one side of ring, and is therefore slightly rotated by an angle $\theta$, in order to have the wafers in the field of view. An electron trajectory will therefore be observed slightly from the side; the electron will not be imaged as a point, but as a line with width $d_{\text {gap }} \times \sin (\theta)$, where $\theta$ for our set-up is $\arctan (D / s)=\arctan (12 \mathrm{~cm} / 5.9 \mathrm{~m})=20 \mathrm{mrad}$. The signal will therefore pick up a horizontal resolution contribution from a light distribution with full width of $5 \mathrm{~cm} \times \sin (20 \mathrm{mrad})=1 \mathrm{~mm}$, corresponding to an rms width of $\sigma_{\text {side }}=1 \mathrm{~mm} / \sqrt{12}=290 \mathrm{um}$. In the horizontal plane we therefore expect a larger resolution than in the vertical plane, on the order of

$$
\sigma_{\text {res,th }, \mathrm{X}}=\sqrt{\sigma_{\text {res,th }}^{2}+\sigma_{\text {side }}^{2}}=362 \mu \mathrm{m}
$$

The effective resolution of the system was measured by comparing the electron beam size measured, with a Kodak lanex screen and a second Cherenkov system. The second Cherenkov system, which we call the "NEAR" lens system, has a smaller air gap and the is lens placed close to the wafers so that the entire Cherenkov ring falls on the lens, corresponding to operation mode 
Calc. light, $15 \mathrm{~cm}$ aperture

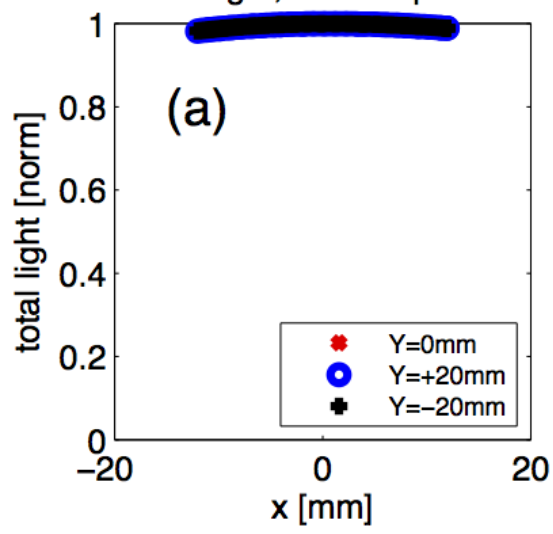

Calc. light, $5 \mathrm{~cm}$ aperture

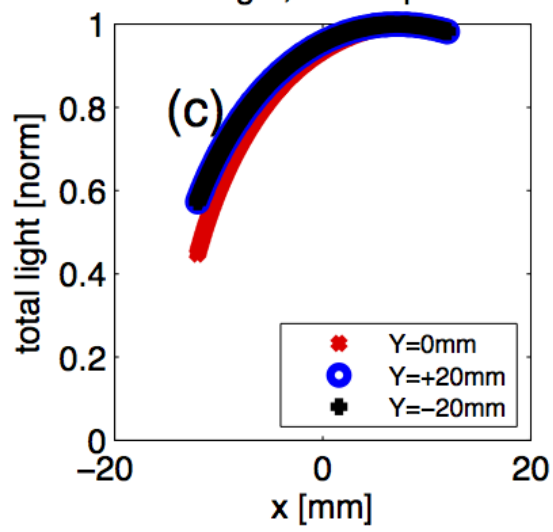

Meas. light, $15 \mathrm{~cm}$ aperture

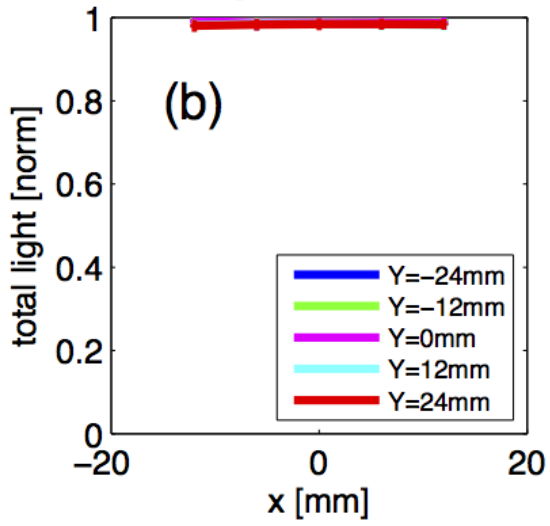

Meas. light, $5 \mathrm{~cm}$ aperture

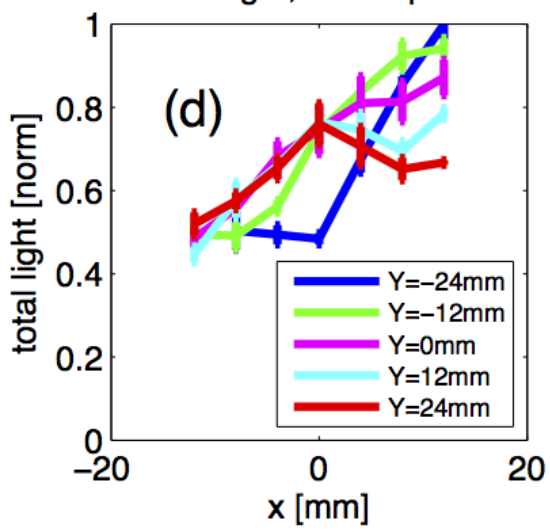

Figure 7: Light yield for the FACET spectrometer profile monitor, as function of horizontal charge position, for a set of vertical positions. The top row of plots shows light yield for the nominal profile monitor configuration, with the lens iris fully open; (a) shows the calculated light yield using Eqs. (3) and (4), (b) shows the measured light yield. We observe less than $3 \%$ variation in the light yield for any horizontal and vertical charge location, both for the calculated case and for the measured case. In comparison, the bottom row of plots shows light yield for a test configuration with the lens iris set to 1/3 of full aperture; (c) shows the calculated light yield using Eqs. (3) and (4), (d) shows the measured light yield. Both the calculated and the measured case show for this configuration a sharp fall-off in the light yield, as function of the horizontal position of the particles. The measured fall-off is in reasonable agreement with the calculated values, and indicates the validity of the ray tracing model. 
"1" in Figure 1. This is as opposed to the main lens system described in this paper, which we call "FAR" for comparison, which operates in mode "2" in Figure 1. The electron beam size at the location of the Cherenkov wafers was varied using focusing magnets. By comparing the spot size on the different systems, the resolution of systems could be estimated. The resolution of the "FAR" system was measured to be

$$
\begin{gathered}
\sigma_{\text {res }, x}=350 \mu \mathrm{m} \pm 25 \mu \mathrm{m} \\
\sigma_{\text {res }, y}=230 \mu \mathrm{m} \pm 20 \mu \mathrm{m}
\end{gathered}
$$

For the "FAR" system, the measured resolution in $y$ is consistent with the estimate given in Eq. (6), and the measured resolution in $x$ is consistent with the estimate given in Eq. (7). In comparison, for the "NEAR" system the resolution in both planes were measured to be

$$
\sigma_{r e s, N E A R}=140 \mu \mathrm{m} \pm 20 \mu \mathrm{m},
$$

which is within the expected resolution from known sources for the "NEAR" system. Figure 8 illustrates the asymmetry in the "FAR" system, by comparing a single electron beam shot, generating light on both systems. On the "NEAR" system, the measured spot size is similar in both planes, while for the "FAR" system, the measured spot size is significantly larger in the horizontal plane than in the vertical plane, as expected from the discussion above.

The main purpose of the FACET Cherenkov profile monitor is to perform spectrometer energy measurements. The dispersion induced by the spectrometer dipole, at the nominal FACET beam energy of $20.35 \mathrm{GeV}$, is $D_{0}=62 \mathrm{~mm}$. We define the detector energy resolution as $\sigma_{\text {res, } E}=$ $\sigma_{r e s, y} / D \times E=\sigma_{r e s, y} / D_{0} \times E^{2} / E_{0}$. For nominal parameters the energy resolution is $\sigma_{\text {res }, y} / D_{0} \times E_{0}=76 \mathrm{MeV}$, or $0.4 \%$ of the nominal energy. The energy resolution scales with the particle energy squared, yielding better resolution at lower energy and poorer resolution for higher energy. Figure 9 displays the energy resolution as function of the particle energy $E$. 

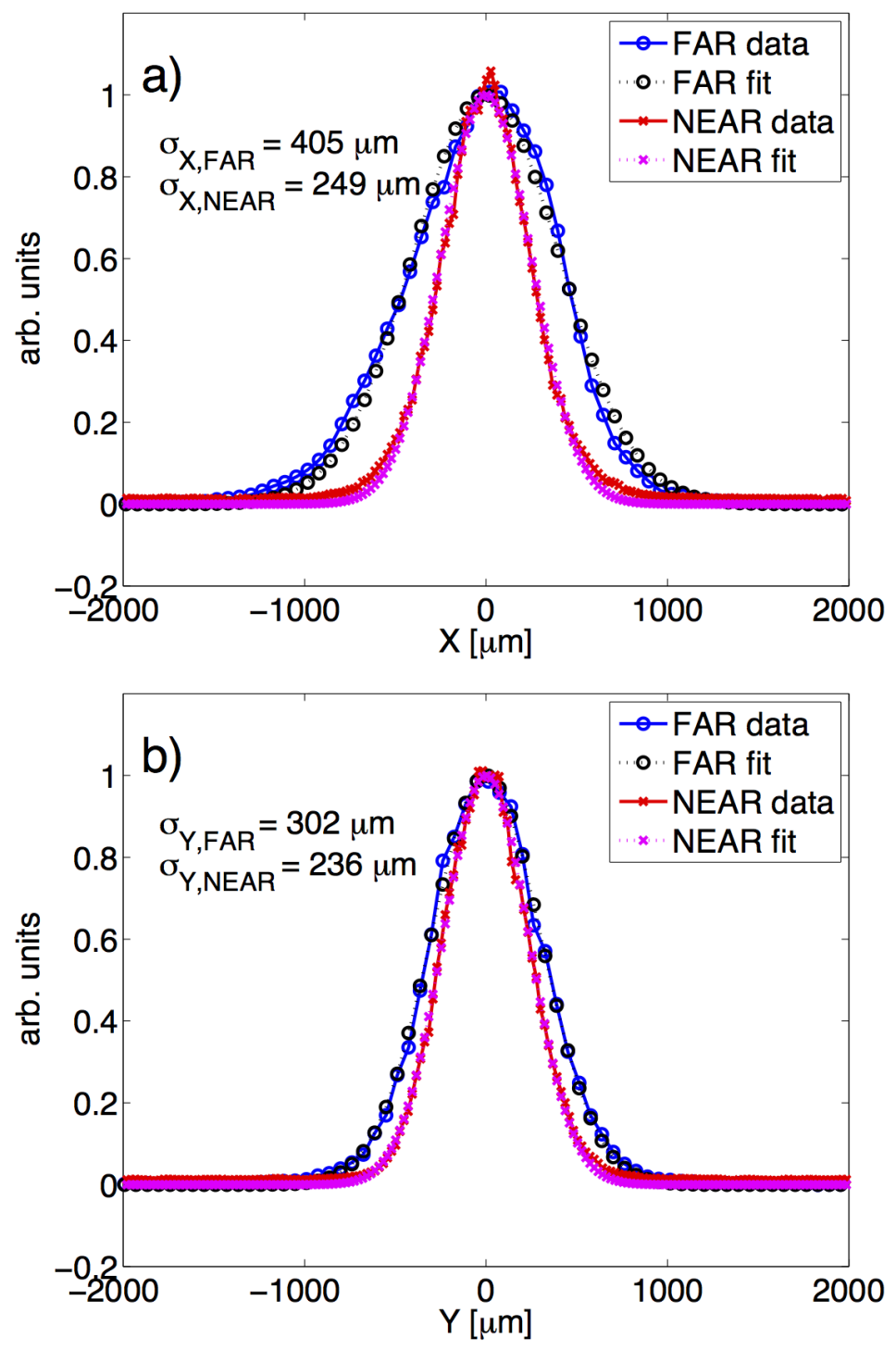

Figure 8: Example of the projected light distribution generated from a single electron shot, in both planes, for both the "FAR" system described in detail in this paper, where only a fraction of the Cherenkov ring hits the lens, and the "NEAR" system where the entire Cherenkov ring hits the lens. On the "NEAR" system, the measured spot size is similar in both planes, while for the "FAR" system, the measured spot size is significantly larger in the horizontal plane than in the vertical plane. For the "FAR" system a larger resolution in the horizontal plane is expected as the camera is viewing the air gap from a slight horizontal angle. 


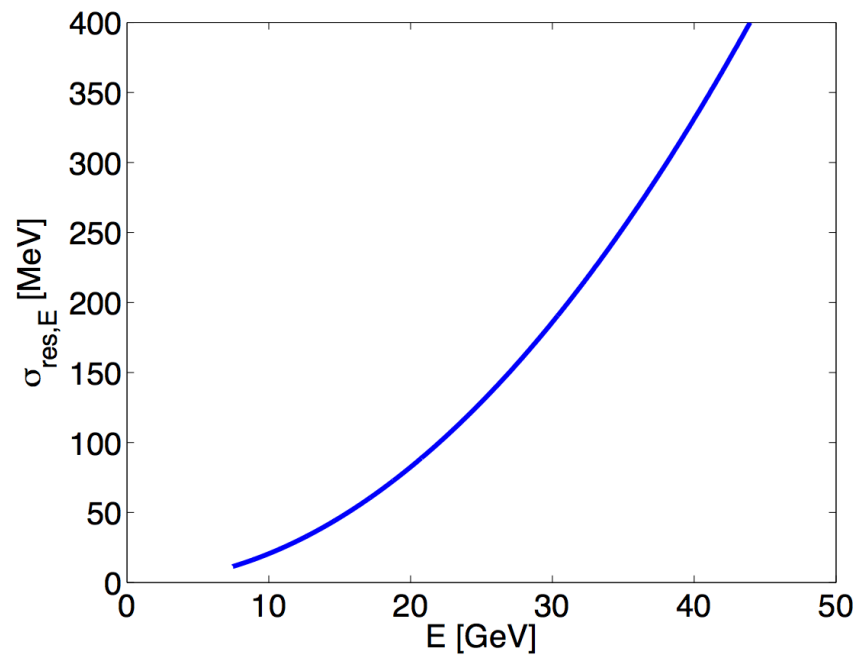

Figure 9: The detector energy resolution of the FACET Cherenkov spectrometer, as function of the particle energy. The energy resolution given here is calculated as the measured spatial resolution in the vertical plane, divided by the dispersion due the spectrometer dipole, times the particle energy. For the nominal FACET beam energy of $20.35 \mathrm{GeV}$, the energy resolution is $76 \mathrm{MeV}$, or $0.4 \%$ of the nominal energy. 


\subsection{Wafer damage}

We have observed that the intense electron beam can create visible damage on the $800 \mu \mathrm{m}$ thick silicon wafers, if enough electron pulses hit the wafers. Since visible damage affects the reflection coefficient unevenly, this may create an error in the measurement.

There will be a temperature increase in the Si wafers due to energy deposition originating from collisional losses of the electron beam in the wafers. The mean collisional losses, $d E / d x_{\text {coll }}$, for a $20 \mathrm{GeV}$ electron passing through Silicon is $2.3 \mathrm{MeV} \mathrm{cm} \mathrm{g}^{-1}$ [16]. We estimate the peak surface density of the FACET electron beam to $\hat{\sigma}_{b}=N /\left(2 \pi \sigma_{x} \sigma_{y}\right)=8 \times 10^{14} \mathrm{~cm}^{-2}[1]$ where $N=2 \times 10^{10}$ is the beam charge and $\sigma_{x}=\sigma_{y}=20 \mu \mathrm{m}$ are the rms spot sizes. We have assumed a Gaussian beam. The specific heat capacity of Silicon at $25^{\circ} \mathrm{C}$ and $1 \mathrm{~atm}$ is $C_{\mathrm{Si}}=0.7 \mathrm{~J} \mathrm{~g}^{-1} \mathrm{~K}^{-1}$ [17]. A single pass of the beam through the wafers may thus lead to a peak temperature increase of $\Delta T=d E / d x_{\text {coll }} \times \hat{\sigma}_{b} / C_{\mathrm{Si}}=418 \mathrm{~K}$. This is a significant temperature increase compared to the melting point of Si which is $1687 \mathrm{~K}$, and may be a cause of the damage observed.

We have investigated the damage quantitatively by measuring the reflectivity of two wafers which have seen a different number of electron pulses in the FACET run of spring 2013. One set of wafers was inserted into the electron beam only when the FACET plasma experiment was running. The experiment had about 150 hours of beam time, running at $1 \mathrm{~Hz}$ for most of the time, and the wafers were exposed to a few times $10^{5}$ pulses of up to $2 \times 10^{10}$ electrons. Figure 10 (upper part) shows the reflection of diffuse light on these wafers after the experiment. No visible damage was observed on this set of wafers. Another set of wafers was inserted into the electron beam when the experimental wafers were extracted, in order to use the Cherenkov monitor during beam commissioning. These wafers were exposed to a few times $10^{7}$ pulses, occurring at up to $10 \mathrm{~Hz}$. Figure 10 (lower part) shows reflection of diffuse light on these wafers after the experiment. There is a clear mark where the beam has hit the wafers, located between pixels 450 and 500 in the $x$-direction. Outside the core of the beam the reflectivity is reduced by up to $30-40 \%$. The system could still be used for beam commissioning purposes even this with damage. 

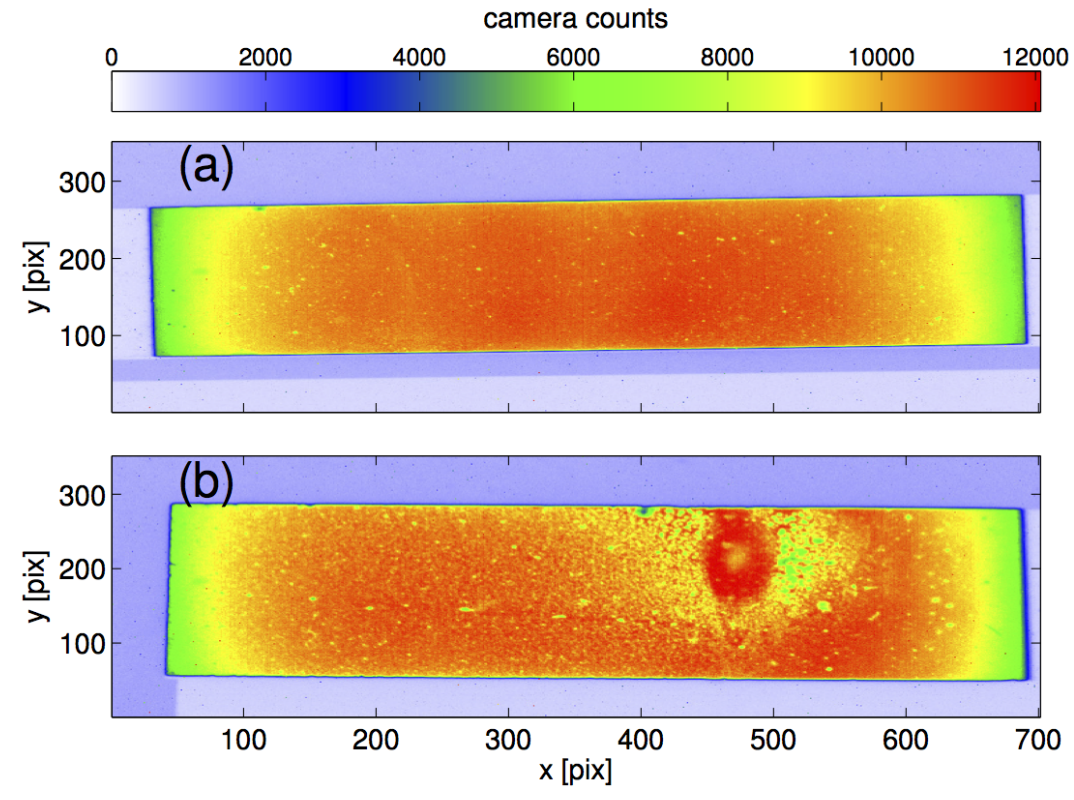

Figure 10: (a) Reflection of diffuse light off silicon wafers used for the FACET experiments in 2013. These wafers were exposed to a few times $10^{5}$ pulses of up to $2 \times 10^{10}$ electrons, and show no visible sign of degradation. (b) Reflection of diffuse light off silicon wafers which have been in the beam line during all of the FACET commissioning in 2013. These wafers were exposed to a few times $10^{7}$ pulses of up to $2 \times 10^{10}$ electrons, and shows significant degradation, which translates to reduced light yield in the affected areas. 


\section{Conclusions}

We have discussed the principle of a transverse beam profile monitor based on Cherenkov light emission in air. The light yield in this type of Cherenkov monitor may be up to 100 times larger than the light yield from optical transition radiation. Other key advantages of the Cherenkov monitor is good robustness to radiation damage, excellent separation of incoming photons and electrons, linearity with beam charge and independence of the signal intensity with particle energy. The depth of field effect may give a limitation on the system resolution compared to a Lanex screen. We have shown that by careful design, a large field of view can be obtained, with very good light coverage in the entire field of view. The system is simple and robust, and easy to install, provided the beam is transported through air or similar media. The silicon wafers used to reflect the Cherenkov light can survive at least $10^{5}$ pulses of up to $2 \times 10^{10}$ electrons without significant degradation. If the silicon wafers are hit by an order of magnitude more electron pulses the wafer reflectivity may be affected, and the wafers should be replaced with a new set for optimum performance.

\section{Acknowledgments}

We are grateful for the cooperation of the FACET operational crew for help with providing the beam used for testing the system. We thank Stanford University students Julien De Mori and Matthew Kahane for help with the installation of the system, and Carl A. Lindstrøm for proofreading. Silicon wafers have been procured from WRS Materials, and we appreciate the excellent service from Michelle Piffero. This work is supported by the Research Council of Norway and the U.S. Department of Energy under contract number DE-AC02-76SF00515.

[1] M. J. Hogan et al., New J. Phys. 12, 055030 (2010)

[2] X. Wang et al., Nature Communications, 10, 1988 (2013)

[3] A Multi-TeV Linear Collider Based on CLIC Technology: CLIC Conceptual Design Report, CERN, Geneva (2012)

[4] I. Blumenfeld et al., Nature 445, 741 (2007)

[5] R. Ischebeck et al., in Proceedings of PAC'07, p. 4168 (2007) 
[6] M. Litos et al., Nature 515, 92 (2014)

[7] R. Carr and H. Wiedemann, in Handbook of Accelerator Physics and Engineering, 3rd ed. World Scientific Publishing Co. Pte. Ltd, 2006, sec. 3.1.6, p. 214.

[8] A. Buck et al., Rev. Sci. Instrum., 81, 033301 (2010)

[9] P. A. Cherenkov, Dok. Akad. Nauk SSSR 2, 451 (1934)

[10] K.P. Birch and M.J. Downs, Metrologia 30, 155 (1993).

[11] K.P. Birch and M.J. Downs, Metrologia 31, 315 (1994).

[12] I. Tamm, J. Phys.(USSR) 1, 439 (1939)

[13] pco.edge 5.5, scientific CMOS camera, http://www.pco.de/categories/scmos-cameras/pcoedge/

[14] USAF Resolution Test Chart, en.wikipedia.org/wiki/1951_USAF_resolution_test_chart

[15] R. Carr and H. Wiedemann, in Handbook of Accelerator Physics and Engineering, 3rd ed. World Scientific Publishing Co. Pte. Ltd, 2006, sec. 3.3.1, p. 243.

[16] W. R. Leo, Techniques for Nuclear and Particle Physics Experiments, Springer (1994).

[17] National Institute of Standards and Technology, http://webbook.nist.gov. 